\title{
LATERAL MENISCUS TEARS IN ACL INJURED KNEE
}

\author{
Anshu Shekhar, Anoop Pilar, Sachin Tapasvi* \\ The Orthopaedic Speciality Clinic, Pune, India.
}

\section{ABSTRACT}

Anterior cruciate ligament (ACL) tears can be associated with injuries to the lateral meniscus (LM) in about 20-30\% cases. The lateral meniscus is more mobile than the medial and besides contributing to load transmission, it also stabilizes the knee in pivot-shift testing. The LM tears more often in the acute setting and its incidence does not rise in cases of chronic ACL instability. Lateral meniscus tears can be minor or major depending how severely the knee function gets impaired. Major tears are the complete radial tears, longitudinal bucket handle tears and posterior root tears. Male gender, high body mass index and contact injury mechanism are all risk factors for an LM tear. Anatomic factors which can contribute to LM tears include a high posterior tibial slope, varus malalignment and greater asymmetry between medial and lateral slopes. The lateral meniscus must be saved and repaired whenever possible to prevent residual knee instability and progressive lateral compartment arthritis, which can set in soon after a meniscectomy.

The development of techniques and technology have rendered most tears amenable to repair. Longitudinal tears can be repaired by the all-inside or inside-out technique and the needles and devices must be inserted through a high anteromedial or transpatellar portal to prevent injury to the popliteal neurovascular structures. A lateral safety incision must always be used for inside-out repairs. Radial tears can be repaired by two horizontal sutures, a cross stich, a cross-tag or a hash-tag suture configuration. Lateral meniscus posterior root repairs are repaired by transtibial technique, either by drilling an independent anatomic tunnel or the sutures pulled out via the ACL tibial tunnel. The lateral meniscus has high healing rates and repairs yield improvement in functional outcome, beside delaying radiographic arthritis.

Keywords: Knee; Lateral Meniscus; Lateral Meniscus Tear; Meniscus Repair; All-inside repair

\section{*Corresponding author:}

Sachin Tapasvi MBBS, MS (Ortho), DNB, FRCS (Glasgow)

Director, The Orthopaedic Speciality Clinic, 16 Status Chambers, 1221/A Wrangler Paranjpe Road, Pune, India.PIN- 411004. Email: stapasvi@gmail.com

Phone No: +919822018871 
The lateral meniscus (LM) is a fibrocartilaginous structure which covers about $80 \%$ of the articular surface of the lateral compartment of the knee. It is circular and inserts anteriorly anterior to the intercondylar eminence adjacent to the broad attachment of the anterior cruciate ligament $(A C L)$ and posteriorly, posterior to lateral tibial spine. The lateral meniscus is loosely attached to the capsular ligament and the posterior horn attaches to the inner aspect of the medial femoral condyle through the anterior and posterior meniscofemoral ligaments ${ }^{1}$. Meniscus has an important role in load transmission as it carries about $40-70 \%$ load across the knee joint, plays a role in shock absorption, proprioception, stability and lubrication of the joint. The LM is much more mobile in the antero-posterior plane and has greater radial displacement than the medial meniscus ${ }^{2}$. The reported prevalence of meniscal injury associated with ACL injury can be as high as $55 \%$ to $72 \%$ and a lateral meniscus tear is more commonly seen in acute injuries ${ }^{3,4}$. LM tears occur in far greater frequency in association with an $\mathrm{ACL}$ injury than as an isolated lesion ${ }^{4}$.

Combined injury of the $A C L$ and $L M$ are significant because they have been shown to induce changes in the articular cartilage geometry of the lateral and medial compartments ${ }^{5}$. A magnetic resonance imaging (MRI) analysis revealed lateral compartment changes like increase in the wedge angle of the posterior horn of the meniscus and posterior-inferior directed slopes of the articular cartilage surface. Medial compartment changes included reduction in the cartilage-to-bone height under the posterior horn of meniscus, decreased cartilage thickness in the posterior regions of the tibial plateau and increased thickness in the mid-region [5]. An ACL and LM injured knee demonstrates greater increase in anterior translation by $2 \mathrm{~mm}$ and increased internal tibial rotation by $3^{0} 6$. Hence, the entire knee joint and not just a single compartment is affected. Another challenge of this injury combination is difficulty in diagnosing on pre-operative MRI scans due to its low sensitivity (0.69), especially when the LM tear is posterior or peripheral ${ }^{7}$. Careful assessment of MRI scan while pre-operative planning is important so as not to miss this injury (Figure 1). Nonetheless, one needs to be prepared to manage this important injury combination in every case of $A C L$ tear based on current evidence for optimal patient outcomes. This narrative review discusses the peculiarities of this injury combination, its causes, risk factors, variations and the current management strategies.

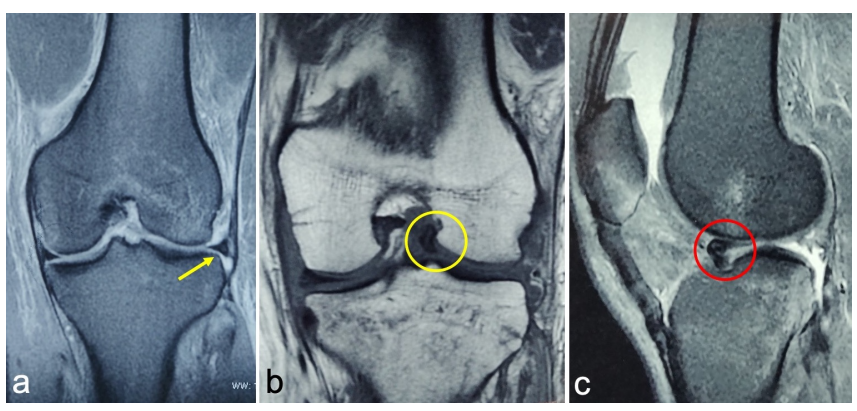

Figure 1: Magnetic Resonance Scan of Lateral Meniscus Tears. (a) Proton density fat-saturated coronal section MRI showing an extruded lateral meniscus (yellow arrow) which is an important indirect sign of a tear. A displaced bucket-handle tear may present as a (b) flipped meniscus lying medially in the intercondylar notch (yellow circle) on T1-weighted coronal image or (c) flipped anteriorly (red circle) as seen on proton density fat-saturated sagittal image. Note the bone marrow edema in the lateral femoral condyle and posterolateral tibia.

\section{Types of Lateral Meniscus Tears:}

The lateral meniscus can tear in any pattern along with an ACL injury. However, some tear patterns are characteristic of this injury combination. Feucht et al have described a classification based on the severity of such tears, based on their morphology ${ }^{8}$. 
Major Tears- Radial tears; Unstable longitudinal tears and Lateral meniscus posterior root tears (LMPRT).

Minor Tears- Incomplete longitudinal tears; Complete but stable longitudinal tears within $1 \mathrm{~cm}$ in front of the popliteus tendon and radial or oblique tears of $<75 \%$ of the width of the meniscus.

The major tears are the ones with grave biomechanical consequences and can cause more severe disruption of knee function if not repaired. The commonest of these have been reported to be the longitudinal vertical tears ${ }^{9}$. However, Krych et al found that the oblique radial tear (Figure 2) was the commonest tear type in their series of 600 patients, comprising $18 \%$ of all meniscus tears ${ }^{10}$. Interestingly, horizontal and complex LMTs are associated with a significantly higher incidence of $>$ grade 2 chondral lesions in the lateral femorotibial compartment, compared to longitudinal or radial tears ${ }^{9}$. Krych et al have also proposed a classification of the lateral meniscus oblique radial tears ${ }^{10}$ described in Table 1.

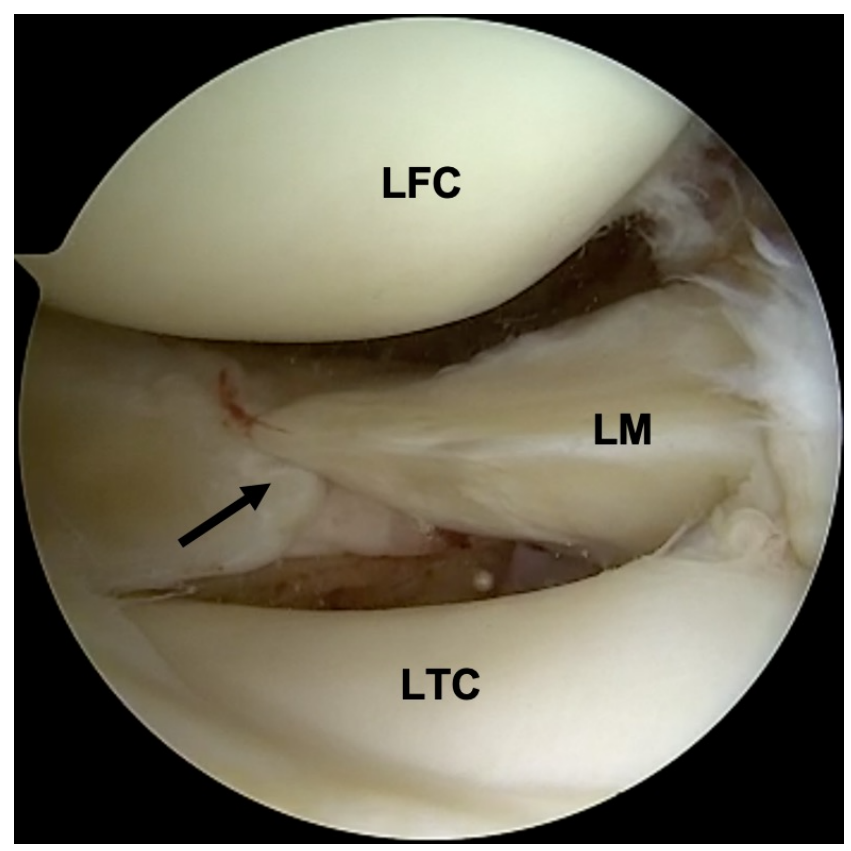

Figure 2: Lateral Meniscus Oblique Radial Tear (Right Knee). An incomplete lateral meniscus radial tears medial to the popliteus hiatus (black arrow). LFC- Lateral femoral condyle, LM- Lateral meniscus, LTC- Lateral tibial condyle.

Lateral meniscus root tears, especially of the posterior root, typically occur in association with $\mathrm{ACL}$ tears. Root tears are those which involve disruption of the bony attachment of the meniscus or radial tears within $10 \mathrm{~mm}$ of this insertion. LMPRTs are far more common than anterior root tears. The mechanism of injury of LMPRT is hypothesized to be a violent anterior translation and external rotation force, causing the lateral quartet' injury pattern. The lateral quartet consists of and $A C L$ tear, lateral meniscus posterior root avulsion and bone bruises in the lateral femoral condyle and posterolateral tibia ${ }^{11}$. The presence of the meniscofemoral ligament (MFL) at the posterior root adds another dimension to the biomechanics, not seen on the medial side. A cadaveric study by Forkel et al found that avulsion of the posterior root with an intact MFL, maintains meniscus function and stabilizes lateral compartment pressure ${ }^{12}$. Forkel et al have also classified LMPRTs in to 3 types based on location and MFL status ${ }^{13}$, described in Table 1:

\section{Table 1: Classification of Lateral Meniscus Tears}

\begin{tabular}{|l|l|}
\hline \multicolumn{2}{|l|}{ Classification of Lateral Meniscus Oblique Radial Tears ${ }^{[10]}$} \\
\hline Type 1 & Partial oblique tear within $10 \mathrm{~mm}$ of root attachment \\
\hline Type 2 & Complete oblique tear within $10 \mathrm{~mm}$ of root attachment \\
\hline Type 3 & Partial oblique tear more than $10 \mathrm{~mm}$ from root attachment \\
\hline Type 4 & $\begin{array}{l}\text { Complete oblique tear more than } 10 \mathrm{~mm} \mathrm{from} \mathrm{root} \\
\text { attachment }\end{array}$ \\
\hline \multicolumn{2}{|l|}{ Classification of Lateral Meniscus Posterior Root Tears ${ }^{[13]}$} \\
\hline Type 1 & Avulsion of the posterior root with intact MFL \\
\hline Type 2 & Complete radial tear between the root attachment and MFL \\
\hline Type 3 & $\begin{array}{l}\text { Avulsion of the posterior root and injury to the meniscal } \\
\text { attachment of MFL }\end{array}$ \\
\hline
\end{tabular}

\section{Incidence and Risk Factors:}

The incidence of LM tear (LMT) in association with $\mathrm{ACL}$ injury has been reported to be about $20 \%$ $31 \%$, although an incidence of $72 \%$ has been reported by Nikolic with recent ACL tears $4,10,14,15$. There are several demographic, anatomic and activity related risk factors attributed to a higher 
incidence of LMTs. In adult patients, their incidence is much higher in acute $A C L$ tears and delaying ACLR beyond 12 months has not been found to significantly increase the Odd's ratio (OR) of LMTs ${ }^{14-16}$. In the study of Kluczynski et al, male gender and injury interval $<6$ weeks was found to be predictive of a lateral meniscus tear ${ }^{17}$. It is postulated that since the lateral meniscus is mobile and does not contribute to knee stability as much as the medial meniscus, it is not under excess stress in an ACL deficient knee ${ }^{9}$. Hence, LMTs usually result from the index trauma and are unlikely to occur later with persistent instability. In Feucht's series, a strong association of 'major' lateral meniscus tears was found with male gender ratio (OR 7.38), age <30 years (OR 5.85) and contact injury mechanism (OR 18.49) ${ }^{8}$. The independent risk factors for occurrence of LMPRTs are participation in contact sports, a concomitant medial meniscus tear and higher body mass index (BMI) ${ }^{18,19}$. Anderson et al studied the incidence and risk factors for meniscus tears in children and adolescents ${ }^{20}$. They found that a younger age, return to sports before surgery and delay of ACLR beyond 6 weeks were significantly co-related with higher occurrence of LMT. Vavken et al have also found a higher BMI and delay in ACLR leading to higher LMTs in pediatric and adolescent patients 21. A lower incidence of lateral meniscus tears in amateur high school female athletes was reported by Piasecki ${ }^{22}$.

Amongst anatomic factors, Mansori et al have reported a greater risk of lateral meniscus tears with high lateral tibial slopes ${ }^{23}$. In a CT scan analysis, Gaillard et al found that a greater anteroposterior length of the lateral femoral condyle with respect to the lateral tibial condyle and lesser convexity of the lateral tibial plateau were significantly associated with LMTs. As such, the lateral compartment of males were at a greater risk ${ }^{24}$. Several anatomic factors have been reported to have an association with LMPRTs. These include greater tibial varus, steep posterior tibial slope and greater asymmetry between lateral and medial slopes ${ }^{19,25}$. Wyatt et al used a community registry to follow patients who had undergone a primary and revision ACLR. They found that the lateral meniscus tears much more frequently in primary $A C L$ tears (32.2\%) than in retears (18.4\%) ${ }^{26}$. A comparison of MOON and MARS study groups also revealed a significantly lower OR of encountering a new lateral meniscus tear in the revision setting ${ }^{27}$.

It is evident from the available literature that most of the risk factors for a LMT in an ACL injured knee are not modifiable. A notable exception are children and adolescents, in whom an early ACLR and not allowing sports before surgery might be protective for sustaining LMTs.

\section{The Case for Saving the Lateral Meniscus:}

The lateral meniscus is important for the knee because of its critical role in knee stability and preserving lateral compartment biomechanics. The lateral meniscus restrains anterior tibial translation in an $\mathrm{ACL}$ deficient knee during combined valgus and rotatory loading, as in a pivot shift manoeuvre ${ }^{28}$. Knees with $\mathrm{ACL}$ tears which have torn lateral meniscus demonstrate greater dynamic postural instability than those with medial meniscus tears 29. A 3D finite-element analysis was performed by Mononen et al to assess the effects of partial lateral meniscectomy on tibial articular surface. They found that the tibial cartilage contact pressures increased by $50 \%$, stresses by $44 \%$, strains by $21 \%$ and pore pressures by $43 \%$. The increase in stress and strains was primarily during the initial half of the gait cycle, i.e. during heel strike and mid-stance ${ }^{30}$. Peña et al performed a finite element analysis of the human tibiofemoral joint to compare the effects of similar amounts of medial and lateral meniscectomy ${ }^{31}$. Under axial compressive femoral loading, the maximum shear stress was $288 \%$ and $323 \%$ higher after a total and partial lateral meniscectomy respectively, compared to similar meniscectomy in the medial compartment. 
These forces are detrimental to the cartilage health and will lead to progressive osteoarthritis in the lateral compartment. Total resection of any portion of the LM has been shown to increase T2 elevation of the articular cartilage of the lateral femoral cartilage on MRI scans after just 6 months 32.

There is plenty of evidence that lateral meniscus deficiency has more profound effects for the lateral compartment than medial meniscus deficiency has for the medial side, in terms of both clinical and radiological outcomes ${ }^{33,34}$. The worse prognosis for the lateral meniscectomy has been seen for a stable knee as well ${ }^{35}$. The incidence of radiographic degenerative changes after an isolated partial lateral meniscectomy can be as high as $84-92.9 \%$ in the long term, where the amount of resection is a determining factor ${ }^{36,37}$. The long-term outcomes of partial lateral meniscectomy are worse in patients who are older than 40 years, have higher $\mathrm{BMI}$, valgus malalignment and cartilage lesions at the time of index surgery ${ }^{38}$. An encouraging result about lateral meniscus repairs is that they have significantly lower failure rates than medial repairs. The re-operation rates are also much lower when a concomitant $A C L$ reconstruction is performed ${ }^{39}$. Based on these consistent findings from literature, there is a compelling case to repair and save as much of the lateral meniscus as is possible in every case of $A C L$ instability.

\section{Management of Specific Tear Patterns:}

The tears of the lateral meniscus can be managed by one of the three methods: meniscectomy, abstention and repair. The importance of preserving LM tissue has been discussed above. Abstention is the practice of leaving stable tears in-situ with or without biological augmentation like rasping or trephination to potentiate healing. The advances in optics, instruments, implants and understanding of knee biomechanics has prompted more tears to be repaired than resected. The challenges specific to performing a lateral meniscus repair are the risk of injury to neurovascular structures, entrapment of the popliteus tendon and poorer capsular tissue to hold the sutures compared to the medial side.

\section{General Principles for Lateral Meniscus Surgery}

- Position: The patient can be placed in the supine flat-table or a hanging leg position. The knee has to be taken to a 'figure-of- 4 ' position with the knee flexed $70-90^{\circ}$ for visualization of the meniscus.

- Exposure: The lateral compartment is not 'tight' like the medial side. Hence, exposure is not a problem usually and is improved by lifting the heel up to distract the lateral side.

- Portals: The standard anterolateral portal is usually adequate as a viewing portal after removal of the fat pad as necessary. The anteromedial portal serves as the working portal, both for performing meniscectomy and repairs. A higher location of the anteromedial portal makes instrumentation easier by providing access above the lateral tibial eminence ${ }^{40}$. However, an accessory anteromedial or central patellar portal maybe required for viewing the more anterior areas of the meniscus, especially for a repair.

- Safety Incision: A safety incision on the lateral side is necessary when performing an inside-out repair. The plane for placing a retractor is between the biceps femoris tendon and iliotibial band superficially, and the capsule and lateral head of gastrocnemius tendon deeper ${ }^{40}$ (Figure 3 ). It helps protect the popliteus neurovascular bundle posteriorly and the common peroneal nerve laterally.

Meniscus Repair techniques: The techniques of allinside, inside-out, outside-in or transtibial repair can all be used for repairing the lateral meniscus, depending on tear location. Generally, tears posterior to the popliteus tendon are repaired 
using all-inside devices, while more anterior tears can be repaired using the inside-out technique and zone-specific cannulae. These two techniques have been found to be comparable, both in terms of clinical failure rates and subjective functional outcomes ${ }^{41}$. It is advised to place sutures adjacent to the popliteus tendon, although placing sutures or all-inside devices through the tendon has not been found to result in adverse outcomes ${ }^{40,42}$. For inside-out repairs, the needles must be inserted from the anteromedial portal only and never from the anterolateral portal because the needle trajectory in such a case will endanger the popliteus neurovascular structures. The transtibial repair is reserved for root tears or posterior radial tears close to the root.

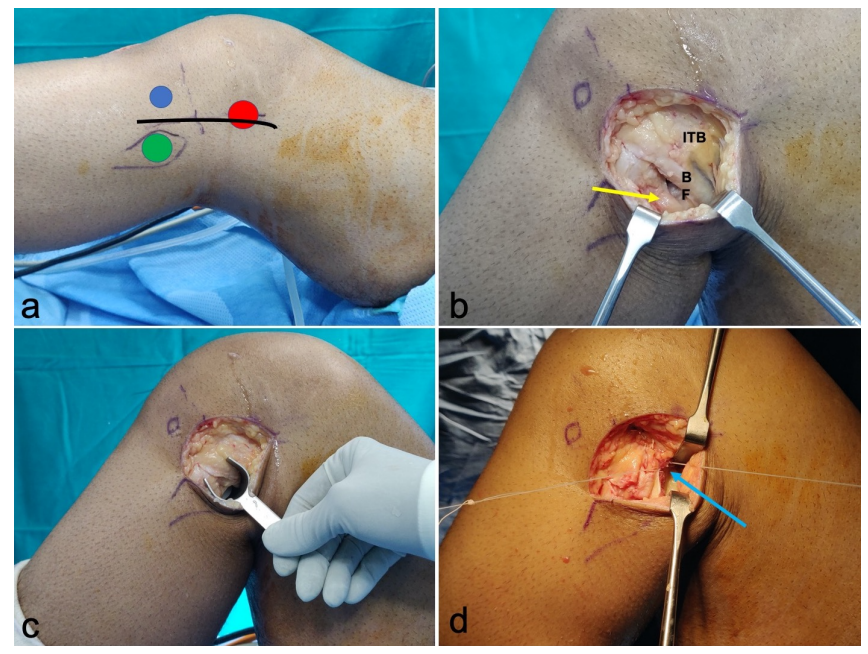

Figure 3: Lateral Safety Incision for Left Knee. (a) Surface landmarks for the skin incision - Fibula head (red dot), Lateral epicondyle (red dot), Gerdy's tubercle (blue dot) and incision marking (black line). (b) The superficial plane is between the iliotibial band (ITB) and biceps femoris tendon (BF) with the common peroneal nerve lying posterolateral to it (yellow arrow). (c) A Henning's retractor placed between the posterolateral capsule and lateral head of gastrocnemius. (d) Inside-out repair sutures exiting in the safe plane can be tied over the capsule (blue arrow) in $90^{\circ}$ knee flexion.

\section{Lateral Meniscus Longitudinal Tears}

The vertical/longitudinal tear is most common type of lateral meniscus tear [9]. This tear can involve any of the three zones and can occur at any location of the meniscus. Further, these tears maybe either stable or unstable on probing during arthroscopy. Lee et al had proposed that stable tears in the posterior horn can be left in-situ and do not propagate to larger tears after about 3 years of follow-up ${ }^{43}$. They found an MRI healing rate of $86 \%$ and complete healing on second-look arthroscopy in $75 \%$ patients, while $18 \%$ had healed partially ${ }^{43}$. Although these are encouraging results, some of these 'conservatively' managed tears do have the potential to propagate. A more recent cadaveric experiment using a robotic testing system found that such stable tears do progress with cyclical loading of the knee when left alone after only 100 cycles of knee loading ${ }^{44}$. This causes the external tibial rotation to significantly increase by up to $45.5 \%$ and the contact forces at the tibiofemoral joint increased by up to $91.9 \%{ }^{44}$. These detrimental effects of a tear progression on knee kinematics and contact forces need to be borne in mind. It is perhaps prudent to be more inclined to repair and save the meniscus, till more data is available as to which subset of patients are unlikely to have a tear propagation.

As discussed above, either the all-inside or insideout technique can be used to repair these tears. A combination of both techniques is required for large bucket-handle repairs (Figure 4). An overarching concern when performing lateral meniscus repairs by either technique is the safety of the neurovascular structures in this area. Tears medial to the popliteus tendon are preferably repaired using an all-inside device since the popliteus neurovascular structures are not safe by using any portal or cannula type [45]. The all-inside device for posterior repairs must not be inserted from the anterolateral portal. The popliteal artery was found to be only $4.7 \pm 2.3 \mathrm{~mm}$ and popliteal 
vein $6.7 \pm 2.9 \mathrm{~mm}$ from the device needle in a cadaveric experiment by Mao ${ }^{46}$. Massey et al recommended insertion of these repair devices from an anteromedial or a transpatellar portal because of the lowest risk of neurovascular penetration ${ }^{47}$. Further, keeping the depth setting at $14 \mathrm{~mm}$ leads to lowest risk of gastrocnemius penetration (10\%) and capsular underpenetration (5\%) ${ }^{47}$. The popliteal vessels and peroneal nerve are relatively safer when the allinside repair is performed at $90^{\circ}$ knee flexion than at lower flexion angles ${ }^{48}$. The lateral geniculate artery is another smaller, albeit important structure that must be protected because it is the major vascular supply to the LM. Chen found that injury to this structure occurred in $72 \%$ cases for mid-third repair and in $63 \%$ cases when the needle was passes across the mid-anterior $1 / 3$ junction of lateral meniscus in embalmed cadavers ${ }^{49}$. Cuéllar et al have reported that both all-inside and insideout repair needles are precariously close to the geniculate artery for tears anterior to the popliteus hiatus. However, inside-out suture knot tying on the capsule carries an additional high risk of obliteration on the artery ${ }^{50}$.

A meta-regression analysis found that when performed along with an ACLR, the all-inside technique has significantly higher clinical failure rates $(16 \%)$ compared to the inside-out technique $(10 \%)^{51}$. The patient-reported outcomes of large vertical bucket-handle meniscus repairs have been found to be satisfactory and comparable to small vertical tear repairs ${ }^{52}$. Uzun et al reported comparable functional outcomes between undisplaced vertical longitudinal and displaced bucket-handle LM tears using the all-inside or hybrid suture repair technique. Smoking was identified as a risk factor for failure, irrespective of tear type ${ }^{53}$. Ahn et al reported the outcomes of 13 bucket-handle LM repairs after a median followup of 4 years ${ }^{54}$. There was significant improvement is Tegner activity levels and Lysholm scores, with all patients achieving pre-injury activity level and no re-operation rates for the meniscus ${ }^{54}$. Based on currently available evidence, it is recommend to repair all LM vertical tears using all-inside devices for tears medial and to the popliteus tendon and all-inside or inside-out technique for repairs anterior to the hiatus.

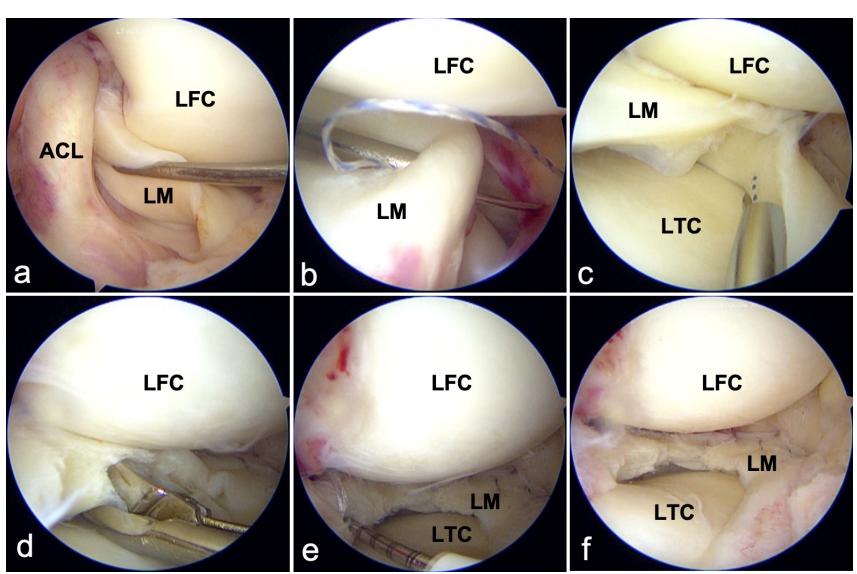

Figure 4: Bucket-handle Lateral Meniscus Repair (Left Knee). (a) The mid-posterior third of the meniscus is torn in the red-red zone and flipped in the intercondylar notch. (b) Repair is begun at the center of the tear by inside-out technique on the superior surface. The zone-specific cannula is inserted from the anteromedial portal. (c) Inferior surface inside-out suture being inserted via the transpatellar portal while viewing from the anteromedial portal. (d) A flap in the white-white zone being excised. (e) Posterior horn being repaired using an all-inside device inserted from the transpatellar portal. (f) The completed lateral meniscus repair has preserved almost the entire tissue. LFC- Lateral femoral condyle, LM- Lateral meniscus, LTC- Lateral tibial condyle, ACL- Anterior cruciate ligament.

\section{Lateral Meniscus Radial Tears:}

The radial LM tear is a devastating injury, especially if it involves the peripheral circumferential fibres which are responsible for maintaining hoop stress in the meniscus. In that scenario, it is akin to a total meniscectomy and leads to complete loss of meniscal function ${ }^{55}$. A 
variant of the classical radial tear, a lateral meniscus oblique radial tear (LMORT) has been described by Krych et al. They reported this to be the commonest LM tear in their series ${ }^{10}$. Considering its challenging nature, a partial meniscectomy was commonly performed for this tear. Bedi et al performed a cadaveric study to assess the dynamic contact mechanics of radial tears in the region of popliteus hiatus ${ }^{56}$. They found that the tears extending up to $60 \%$ of the meniscus width did not lead to change in pressure magnitude or location. However, tears involving $>90 \%$ increased peak pressure in the posteriorperipheral position of the tibia, along with a reduction in contact area. Repair of this tear significantly reduced the peak pressure, but there was no change in contact area. A partial meniscectomy resulted in changes similar to a $90 \%$ tear. Krych et al have proposed partial meniscectomy only for incomplete tears that do not involve the peripheral rim, when present within $10 \mathrm{~mm}$ of the posterior root attachment ${ }^{10}$. Radial LM repair has also been shown to reduce meniscus extrusion, without reducing the dynamic meniscus extrusion of the $L M$, thus preserving it normal mechanics ${ }^{57}$.

Radial tears are challenging to repair. Any technique of repair, i.e. all-inside, inside-out or outside-in can be used depending on the location of the tear. Although the avascular zone of the LM maybe excised, it is prudent to preserve as much meniscus tissue as possible. The inside-out technique is generally considered the 'gold standard' of meniscus repairs. However the superiority of inside-out over all-inside repairs for mid-body radial tears could not be established in a meta-analysis. The load to failure, displacement or site of failure were not different between the two groups ${ }^{58}$. Several techniques of radial tear repair have been described (Figure 5). The simplest of these is the horizontal mattress configuration with two or three sutures (Figure 6). The problem of this technique is that sutures are placed parallel to the collagen orientation in the meniscus. Matsubara described the cross-stitch technique where sutures are placed in an oblique plane with respect to the meniscus collagen ${ }^{59}$. This construct was found to have a significantly higher ultimate failure load, greater stiffness and lower displacement on application of cyclical loads, compared to two horizontal sutures ${ }^{59}$. Stender et al described two novel suture configurationshashtag and crosstag ${ }^{60}$. In these techniques the horizontal and cross stiches were reinforced with a vertical suture on each side of the repair, much like a rebar ${ }^{61}$. Although these configurations were not superior to cross-stitch technique for load to failure or stiffness, they demonstrated significantly less displacement on cyclic loading. The hashtag was superior to the crosstag in resisting displacement ${ }^{60}$. Massey et al reported the superiority of the hashtag or rebar repair over the horizontal or cross-suture in terms of higher load to failure and lower rate of suture cut-out ${ }^{61}$. In more complex tears, especially those close to the root attachment, a hybrid repair technique maybe suitable (Figure 7).

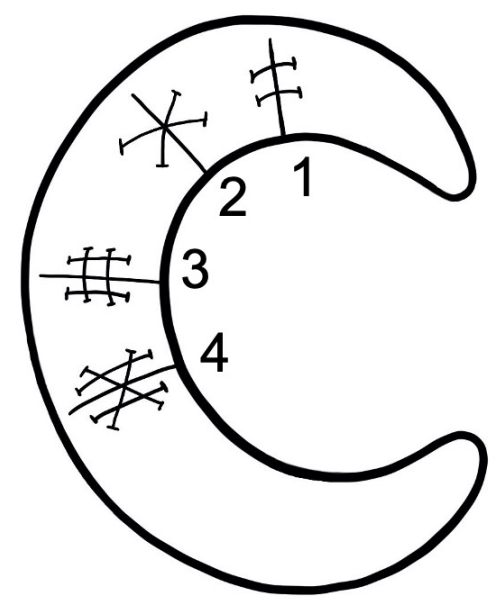

Figure 5: Radial Tears Suture Configurations. The radial tear can be repaired using suture configurations such as the horizontal stitch (1), the cross stitch (2), the cross-tag (3) or the hash-tag (4). 


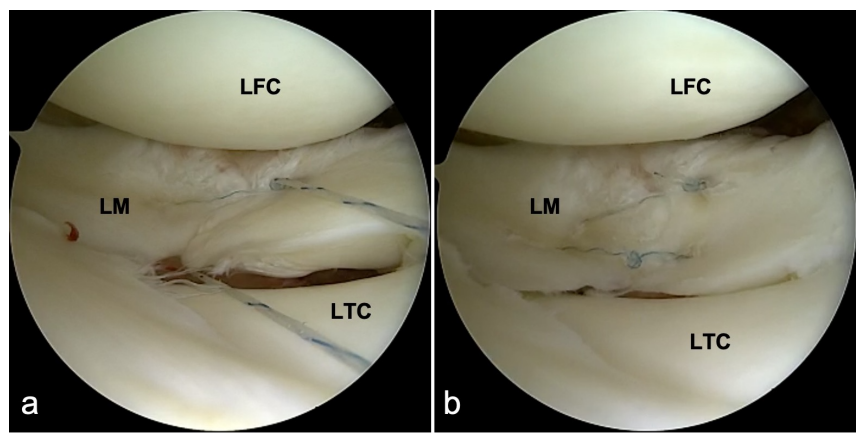

Figure 6: Horizontal Suture Repair of Radial Tear (Right Knee). (a) Sutures placed in a horizontal fashion in the superior and inferior surfaces of the meniscus with an all-inside device. (b) Repair is completed by placing a third suture on superior surface. This is the same tear as in Figure 2. LFCLateral femoral condyle, LM- Lateral meniscus, LTC- Lateral tibial condyle.

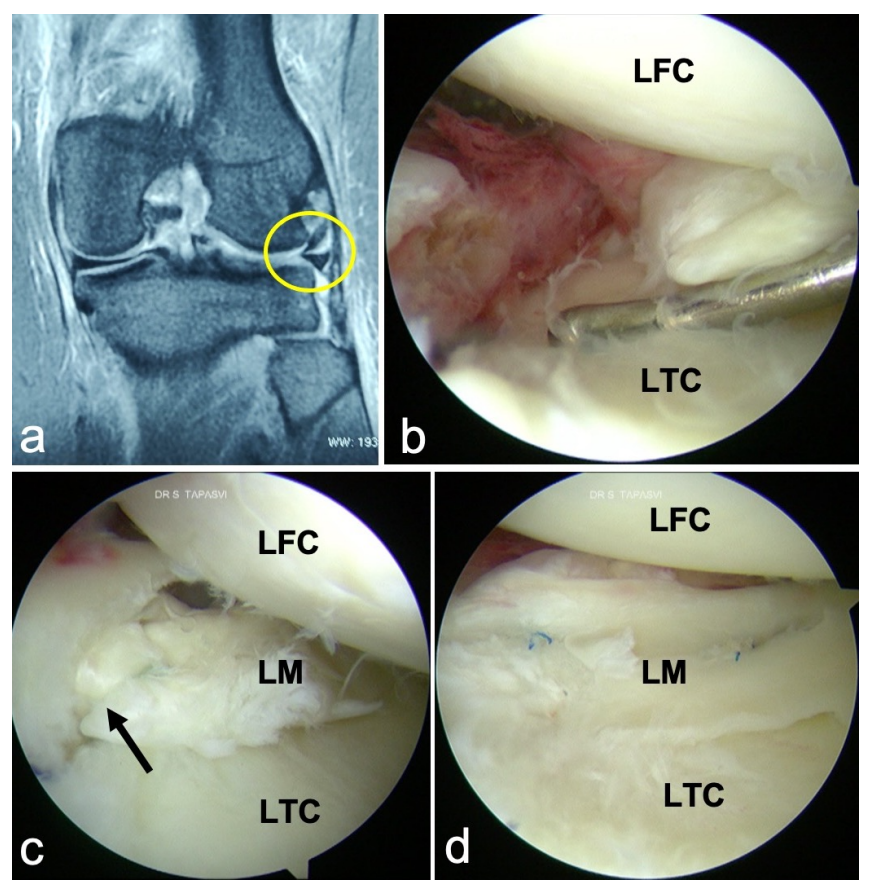

Figure 7: Hybrid Repair of Radial Tear (Left Knee).

(a) Proton density fat-saturated coronal section MRI showing an extruded lateral meniscus and flipped segment of the radial tear (yellow circle) (b) Arthroscopic view of the tear lying within 10 $\mathrm{mm}$ of the posterior root attachment with capsular detachment of the lateral portion. (c) The lateral torn segment is first reattached by $a$ transtibial technique (black arrow) as the medial tissue is insufficient to perform a side-side repair (d). The repair is completed by suturing the posterior horn to the capsule with all-inside devices. LFC- Lateral femoral condyle, LM- Lateral meniscus, LTC- Lateral tibial condyle.

The outcomes of radial LM repairs have also been published. In Tsuji's series of 41 patients, radial tear repairs with an ACLR were compared to isolated ACLR knees after means 3.4 years of surgery. There was no radiographic deterioration in lateral compartment or coronal plane meniscus extrusion as seen on MRI in the radial tear group. However, sagittal meniscus extrusion was significantly greater. Healing was assessed on second-look arthroscopy where $60 \%$ menisci had completely healed, $30 \%$ partially healed and $10 \%$ unhealed. Chondral changes in the lateral tibial plateau had significantly worsened over this period ${ }^{62}$. Wu et al performed a propensitymatched analysis of 18 radial and bucket handle meniscus repairs. There was no difference in the re-operation rates at 2 years and 5 years and the VAS, IKDC and Tegner scores were similar both the groups of patients ${ }^{63}$. Repairing radial tears results in satisfactory healing rates without serious complications and improvements in patient outcomes in the short-term. The sustenance od the results in not known over the long-term ${ }^{64}$.

\section{Lateral Meniscus Root Tears:}

The lateral meniscus posterior root tear (LMPRT), especially those involving the MFL, cause complete dysfunction of the meniscus function. Transtibial repair has been shown to reduce peak contact pressure in the lateral compartment to near normal levels. The contact area also increases compared to a torn state, but is still lower than normal levels ${ }^{65}$. The knee stability in an $A C L$ reconstructed knee is improved significantly after a transtibial root repair on anterior loading and simulated pivot-shift. The ACL graft forces are also reduced close to the native state on anterior translational loading ${ }^{66}$. Thus, a root repair is not 
only protective of the cartilage in the lateral compartment but also shields the ACL graft from excessive forces.

A $2 \mathrm{~mm}$ braided suture tape has been shown to yield a higher maximum failure load compared to a No. 2 suture for root repairs ${ }^{67}$. Due to its close proximity to the $A C L$ tibial attachment, sutures through the root can be pulled down from the $A C L$ tibial tunnel or the posterolateral tunnel in case of a double-bundle reconstruction. This technique has been found to be equivalent to an anatomic re-attachment and normalizes tibio-femoral contact pressures ${ }^{12,68}$. An independent tunnel drilling at the anatomic root insertion is technically challenging but possible and is our preferred technique whenever possible (Figure 8). Some degree of tunnel coalition is common and it is safer to pull the root sutures after $A C L$ graft passage, posterior to the graft. Forkel's Type 2 tears can be repaired by side-to-side suture repair technique, much like the horizontal repair technique for radial tears ${ }^{13}$.

The purpose of a LMPRT repair is to restore meniscus function and prevent lateral arthritis. A retrospective comparative study of 62 LMPRTs by Pan et al reported higher, but statistically insignificant IKDC and Lysholm scores in those who underwent a repair versus those that were not. However, after minimum 2 years follow-up, a higher rate of radiographic arthritis was seen in those whose LMPRT was not repaired ${ }^{69}$. Shelbourne et al also reported that subjective outcomes after mean 10 years were not significantly different in patients whose LMPRT was not repaired compared to a control group with intact posterior roots ${ }^{70}$. In this study also, patients with unrepaired LMPRTs had a significantly greater reduction in lateral joint space. In Anderson's series of 24 patients, 22 lateral meniscus root repairs were functioning successfully after mean 58.6 months follow-up ${ }^{71}$. Ahn et al also reported significant improvement in IKDC and Lysholm scores after all-inside side-to- side suture repair in all 25 patients at mean 18 months post-surgery. MRI scan showed that the sagittal extrusion had reduced significantly after the repair. Second-look arthroscopy in 9 patients found complete healing in 8 and incompletely healed but stable meniscus in one patient ${ }^{72}$.

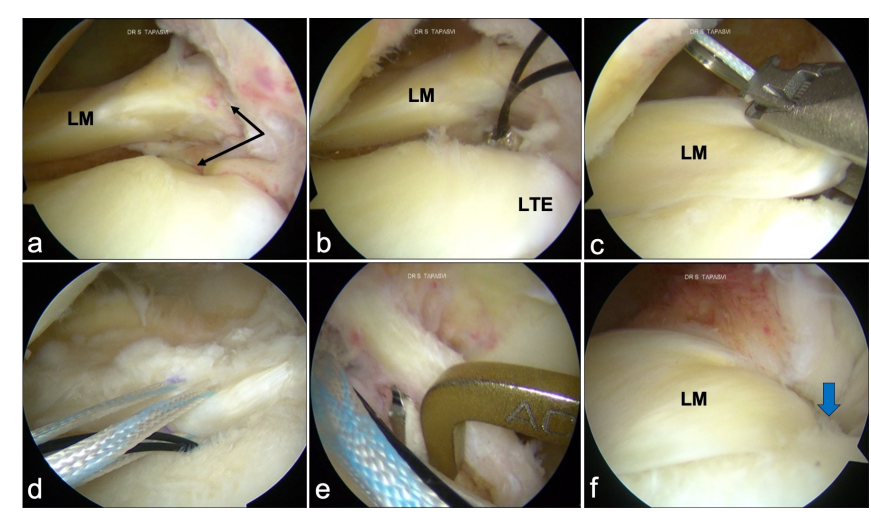

Figure 8: Lateral Meniscus Posterior Root Repair (Right Knee). (a) A complete lateral root tear with avulsion from the tibia (black arrow) and torn meniscofemoral ligament. (b) Transtibial tunnel at the anatomic root attachment and a suture loop passed for retrieving the root sutures. (c) High strength suture braided tape passed through the root. (d) Two tapes are passed which can provide secure reduction and fixation of the meniscus root. (e) A $2.4 \mathrm{~mm}$ drill-tip passing pin drilled at the $A C L$ tibial footprint which is away from the root tunnel. (f) The posterior root is reduced by pulling the tapes through the tunnel (blue arrow). LFCLateral femoral condyle, LM- Lateral meniscus, LTC- Lateral tibial condyle.

\section{Impact on Lateral Meniscus Surgery on Rehabilitation:}

There is no consensus on the type of rehabilitation protocol in terms of weight bearing, bracing or range of motion (ROM) to be followed after a lateral meniscus repair ${ }^{73}$. In fact, the rehabilitation is dependent of several factors like the tear type location, type, repair strength and patient profile. An earlier functional rehabilitation without any restrictions can be instituted after a partial meniscectomy ${ }^{74}$. Similarly, small stable LM 
repairs do not need limitations of weight bearing or ROM and they can rehabilitate in a similar manner as for an isolated ACLR. Early ROM and full weight bearing have not been proven to detrimental to meniscal repairs in systematic reviews ${ }^{75,76}$. However, certain LM repairs such as radial, bucket handle or root require protection in the early phase. A period of 3-4 weeks of protected bearing for these repairs is reasonable although not proven. Delay in return to work and sports is also expected in this scenario and the patient must be counselled regarding the same ${ }^{73}$.

\section{Conclusion}

Lateral meniscus tears are common with acute $\mathrm{ACL}$ tears. The lateral meniscus aids in stabilizing the knee, especially during the pivotshift maneuver. The lateral meniscus must be thoroughly inspected and probed in its entirety in every case of $A C L$ instability, irrespective of the MRI findings. It is worthwhile to repair every lateral meniscus and especially radial, posterior root or large vertical tears.

\section{References}

1. Fox AJ, Bedi A, Rodeo SA. The basic science of human knee menisci: structure, composition, and function. Sports Health. 2012;4(4):340-351. doi:10.1177/1941738111429419

2. Vedi V, Williams A, Tennant SJ, Spouse E, Hunt DM, Gedroyc WM. Meniscal movement. An in-vivo study using dynamic MRI. J Bone Joint Surg Br. 1999 Jan;81(1):37-41. doi: 10.1302/0301620x.81b1.8928.

3. Feucht MJ, Bigdon S, Bode G, Salzmann GM, Dovi-Akue D, Südkamp NP, Niemeyer $P$. Associated tears of the lateral meniscus in anterior cruciate ligament injuries: risk factors for different tear patterns. J Orthop
Surg Res. 2015 Mar 18;10:34. doi: 10.1186/s13018-015-0184-x.

4. Nikolić DK. Lateral meniscal tears and their evolution in acute injuries of the anterior cruciate ligament of the knee. Arthroscopic analysis. Knee Surg Sports Traumatol Arthrosc. 1998;6(1):26-30. doi: $10.1007 / \mathrm{s} 001670050068$.

5. Beynnon BD, Fiorentino N, Gardner-Morse M, Tourville TW, Slauterbeck JR, Sturnick DR, Argentieri EC, Imhauser CW. Combined Injury to the $A C L$ and Lateral Meniscus Alters the Geometry of Articular Cartilage and Meniscus Soon After Initial Trauma. J Orthop Res. 2020 Apr;38(4):759-767. doi: 10.1002/jor.24519.

6. Hosseini A, Li JS, Gill TJ 4th, Li G. Meniscus Injuries Alter the Kinematics of Knees With Anterior Cruciate Ligament Deficiency. Orthop J Sports Med. 2014 Aug 21;2(8):2325967114547346. doi: $10.1177 / 2325967114547346$.

7. De Smet AA, Graf BK. Meniscal tears missed on $M R$ imaging: relationship to meniscal tear patterns and anterior cruciate ligament tears. $A J R A m$ J Roentgenol. 1994 Apr;162(4):905-11. doi: 10.2214/ajr.162.4.8141016.

8. Feucht MJ, Bigdon S, Bode G, Salzmann GM, Dovi-Akue D, Südkamp NP, Niemeyer $P$. Associated tears of the lateral meniscus in anterior cruciate ligament injuries: risk factors for different tear patterns. J Orthop Surg Res. 2015 Mar 18;10:34. doi: 10.1186/s13018-015-0184-x.

9. Beldame J, Wajfisz A, Lespagnol F, Hulet C, Seil R; French Arthroscopy Society. Lateral meniscus lesions on unstable knee. Orthop Traumatol Surg Res. 2009 Dec;95(8 Suppl 1):S65-9. doi: 10.1016/j.otsr.2009.09.007.

10. Krych AJ, LaPrade MD, Cook CS, Leland D, Keyt LK, Stuart MJ, Smith PA. Lateral Meniscal Oblique Radial Tears Are Common With ACL Injury: A Classification 
System Based on Arthroscopic Tear Patterns in 600 Consecutive Patients. Orthop J Sports Med. 2020 May 19;8(5):2325967120921737. doi: $10.1177 / 2325967120921737$.

11. Forkel P, Petersen W. Knee ligment injuries. In: $\mathrm{H}-\mathrm{J}$ Oestern, et al (eds), Bone and joint injuries, European Manual of Medicine. doi:10.1007/978-3-642-383885_24

12. Forkel $P$, Herbort $M$, Sprenker F, Metzlaff $S$, Raschke $M$, Petersen $W$. The biomechanical effect of a lateral meniscus posterior root tear with and without damage to the meniscofemoral ligament: efficacy of different repair techniques. Arthroscopy. 2014;30(7):833840. doi:10.1016/j.arthro.2014.02.040

13. Forkel P, Reuter S, Sprenker F, Achtnich A, Herbst E, Imhoff A, Petersen W. Different patterns of lateral meniscus root tears in ACL injuries: application of a differentiated classification system. Knee Surg Sports Traumatol Arthrosc. 2015 Jan;23(1):112-8.

14. Chen KH, Chiang ER, Wang HY, Ma HL. Correlation of Meniscal Tear with Timing of Anterior Cruciate Ligament Reconstruction in Patients without Initially Concurrent Meniscal Tear. J Knee Surg. 2019 Nov;32(11):1128-1132. doi: 10.1055/s0038-1675783.

15. Keyhani S, Esmailiejah AA, Mirhoseini MS, Hosseininejad SM, Ghanbari N. The Prevalence, Zone, and Type of the Meniscus Tear in Patients with Anterior Cruciate Ligament ( $A C L$ ) Injury; Does Delayed ACL Reconstruction Affects the Meniscal Injury?. Arch Bone Jt Surg. 2020;8(3):432-438.

16. Brambilla L, Pulici L, Carimati G, Quaglia A, Prospero E, Bait C, Morenghi E, Portinaro N, Denti M, Volpi P. Prevalence of Associated Lesions in Anterior Cruciate Ligament Reconstruction: Correlation With
Surgical Timing and With Patient Age, Sex, and Body Mass Index. Am J Sports Med. 2015 Dec;43(12):2966-73. doi: $10.1177 / 0363546515608483$.

17. Kluczynski MA, Marzo JM, Bisson $\amalg$. Factors associated with meniscal tears and chondral lesions in patients undergoing anterior cruciate ligament reconstruction: a prospective study. Am J Sports Med. 2013 Dec;41(12):2759-65. doi: $10.1177 / 0363546513503448$.

18. Praz C, Vieira TD, Saithna A, et al. Risk Factors for Lateral Meniscus Posterior Root Tears in the Anterior Cruciate LigamentInjured Knee: An Epidemiological Analysis of 3956 Patients From the SANTI Study Group. Am J Sports Med. 2019;47(3):598605. doi:10.1177/0363546518818820

19. Okoroha KR, Patel RB, Kadri O, et al. Abnormal tibial alignment is a risk factor for lateral meniscus posterior root tears in patients with anterior cruciate ligament ruptures. Knee Surg Sports Traumatol Arthrosc. 2019;27(2):590-595. doi:10.1007/s00167-018-5171-4

20. Anderson AF, Anderson CN. Correlation of meniscal and articular cartilage injuries in children and adolescents with timing of anterior cruciate ligament reconstruction. Am J Sports Med. 2015 Feb;43(2):275-81. doi: 10.1177/0363546514559912.

21. Vavken P, Tepolt FA, Kocher MS. Concurrent Meniscal and Chondral Injuries in Pediatric and Adolescent Patients Undergoing ACL Reconstruction. J Pediatr Orthop. 2018 Feb;38(2):105-109. doi: 10.1097/BPO.0000000000000777.

22. Piasecki DP, Spindler KP, Warren TA, Andrish JT, Parker RD. Intraarticular injuries associated with anterior cruciate ligament tear: findings at ligament reconstruction in high school and recreational athletes. An analysis of sexbased differences. Am J Sports Med. 2003 
Jul-Aug;31(4):601-5.

doi: 10.1177/03635465030310042101.

23. Mansori AE, Lording T, Schneider A, Dumas $R$, Servien $E$, Lustig $S$. Incidence and patterns of meniscal tears accompanying the anterior cruciate ligament injury: possible local and generalized risk factors. Int Orthop. 2018 Sep;42(9):2113-2121. doi: 10.1007/s00264-018-3992-x.

24. Gaillard R, Magnussen R, Batailler C, Neyret $P$, Lustig $S$, Servien E. Anatomic risk factor for meniscal lesion in association with ACL rupture. J Orthop Surg Res. 2019 Jul 30;14(1):242. doi: 10.1186/s13018019-1281-z.

25. Kolbe $R$, Schmidt-Hebbel $A$, Forkel $P$, Pogorzelski J, Imhoff AB, Feucht MJ. Steep lateral tibial slope and lateral-to-medial slope asymmetry are risk factors for concomitant posterolateral meniscus root tears in anterior cruciate ligament injuries. Knee Surg Sports Traumatol Arthrosc. 2019;27(8):2585-2591. doi:10.1007/s00167-018-5279-6

26. Wyatt RW, Inacio MC, Liddle KD, Maletis GB. Prevalence and Incidence of Cartilage Injuries and Meniscus Tears in Patients Who Underwent Both Primary and Revision Anterior Cruciate Ligament Reconstructions. Am J Sports Med. 2014 Aug;42(8):1841-6. doi: 10.1177/0363546514536020.

27. Borchers JR, Kaeding CC, Pedroza AD, Huston $L$, Spindler KP, Wright RW; MOON Consortium and the MARS Group. Intraarticular findings in primary and revision anterior cruciate ligament reconstruction surgery: a comparison of the MOON and MARS study groups. Am J Sports Med. 2011 Sep;39(9):1889-93.

doi: 10.1177/0363546511406871.

28. Musahl V, Citak M, O'Loughlin PF, Choi D, Bedi A, Pearle AD. The effect of medial versus lateral meniscectomy on the stability of the anterior cruciate ligamentdeficient knee. Am J Sports Med. 2010 Aug;38(8):1591-7. doi: 10.1177/0363546510364402.

29. Lee JH, Lee DH, Park JH, Suh DW, Kim E, Jang KM. Poorer dynamic postural stability in patients with anterior cruciate ligament rupture combined with lateral meniscus tear than in those with medial meniscus tear. Knee Surg Relat Res. 2020 Jan 1;32(1):8. doi: 10.1186/s43019-019-0027$\mathrm{x}$.

30. Mononen ME, Jurvelin JS, Korhonen RK. Effects of radial tears and partial meniscectomy of lateral meniscus on the knee joint mechanics during the stance phase of the gait cycle--A 3D finite element study. J Orthop Res. 2013 Aug;31(8):120817. doi: 10.1002/jor.22358.

31. Peña $E$, Calvo B, Martinez MA, Palanca $D$, Doblaré $\mathrm{M}$. Why lateral meniscectomy is more dangerous than medial meniscectomy. A finite element study. J Orthop Res. 2006 May;24(5):1001-10. doi: 10.1002/jor.20037.

32. Murakami K, Arai Y, Ikoma K, Kato K, Inoue $\mathrm{H}$, Nakagawa S, Fujii Y, Ueshima K, Fujiwara $\mathrm{H}$, Kubo T. Total resection of any segment of the lateral meniscus may cause early cartilage degeneration: Evaluation by magnetic resonance imaging using $\mathrm{T} 2$ mapping. Medicine (Baltimore). 2018 Jun;97(23):e11011. doi: 10.1097/MD.0000000000011011.

33. Salata MJ, Gibbs AE, Sekiya JK. A systematic review of clinical outcomes in patients undergoing meniscectomy. Am J Sports Med. 2010 Sep;38(9):1907-16. doi: 10.1177/0363546510370196.

34. Monllau J.C., Hulet C., de Chou E.., Rochcongar G. (2016) Results of Lateral Meniscectomy. In: Hulet C., Pereira H., Peretti G., Denti M. (eds) Surgery of the Meniscus. Springer, Berlin, Heidelberg. 
https://doi.org/10.1007/978-3-662-

49188-1_34

35. Chatain F, Adeleine $P$, Chambat $P$, Neyret $P$; Société Française d'Arthroscopie. A comparative study of medial versus lateral arthroscopic partial meniscectomy on stable knees: 10-year minimum follow-up. Arthroscopy. 2003 Oct;19(8):842-9. doi: 10.1016/s0749-8063(03)00735-7.

36. Scheller G, Sobau C, Bülow JU. Arthroscopic partial lateral meniscectomy in an otherwise normal knee: Clinical, functional, and radiographic results of a long-term follow-up study. Arthroscopy. 2001 Nov-Dec;17(9):946-52. doi: 10.1053/jars.2001.28952.

37. Bonneux I, Vandekerckhove B. Arthroscopic partial lateral meniscectomy long-term results in athletes. Acta Orthop Belg. 2002 Oct;68(4):356-61.

38. Hulet $C$, Menetrey J, Beaufils $P$, Chambat $P$, Djian P, Hardy P, Potel JF, Servien E, Seil R; French Arthroscopic Society (SFA). Clinical and radiographic results of arthroscopic partial lateral meniscectomies in stable knees with a minimum follow up of 20 years. Knee Surg Sports Traumatol Arthrosc. 2015 Jan;23(1):225-31. doi: 10.1007/s00167-014-3245-5.

39. Ronnblad E, Barenius B, Engstrom B, Eriksson K. Predictive Factors for Failure of Meniscal Repair: A Retrospective DualCenter Analysis of 918 Consecutive Cases. Orthop J Sports Med. 2020 Mar 27;8(3):2325967120905529. doi: $10.1177 / 2325967120905529$.

40. Bonner KF. Meniscus repair: Inside-out suture technique. In: Jackson DW, editor. Master techniques in orthopaedic surgery: Reconstructive knee surgery. Ed 3. Philadelphia: Lippincott, Williams \& Wilkins; 2008:71-88.

41. Grant JA, Wilde J, Miller BS, Bedi A. Comparison of inside-out and all-inside techniques for the repair of isolated meniscal tears: a systematic review. Am J Sports Med. 2012 Feb;40(2):459-68. doi: 10.1177/0363546511411701.

42. Ouanezar $H$, Blakeney WG, Latrobe $C$, Saithna A, Fernandes LR, Delaloye JR, Thaunat $M$, Sonnery-Cottet $B$. The popliteus tendon provides a safe and reliable location for all-inside meniscal repair device placement. Knee Surg Sports Traumatol Arthrosc. 2018 Dec;26(12):3611-3619. doi: 10.1007/s00167-018-4889-3.

43. Lee DW, Jang HW, Lee SR, Park JH, Ha JK, Kim JG. Clinical, radiological, and morphological evaluations of posterior horn tears of the lateral meniscus left in situ during anterior cruciate ligament reconstruction. Am J Sports Med. 2014 Feb;42(2):327-35. doi: 10.1177/0363546513508374.

44. Novaretti J, Herbst E, Chan C, Debski R, Musahl V. Small Lateral Meniscus Tears Propagate and are Detrimental to Knee Kinematics and Contact Forces. Orthop $\mathrm{JI}$ of Sports Med. June 2019. doi:10.1177/2325967119S00238

45. Gupta H, Ghasi RG, Kataria H, Jain V, Shankar V, Daripa RK, Upadhyay AD. Popliteal neurovascular bundle is safe during inside-out repair of medial meniscus without a safety incision. Knee Surg Sports Traumatol Arthrosc. 2019 Jan;27(1):153-165. doi: 10.1007/s00167018-5060-х.

46. Mao DW, Upadhyay U, Thalanki S, Lee DYH. All-Inside Lateral Meniscal Repair via Anterolateral Portal Increases Risk of Vascular Injury: A Cadaveric Study. Arthroscopy. 2020 Jan;36(1):225-232. doi: 10.1016/j.arthro.2019.07.023

47. Massey P, Parker D, Feibel B, Ogden A, Robinson J, Barton RS. Proximity of the Neurovascular Bundle During Posterior- 
Lateral Meniscal Repair: A Comparison of the Transpatellar, Anteromedial, and Anterolateral Portals. Arthroscopy. 2019 May;35(5):1557-1564. doi: 10.1016/j.arthro.2018.12.004.

48. Cuéllar A, Cuéllar R, Cuéllar A, GarciaAlonso I, Ruiz-Ibán MA. The Effect of Knee Flexion Angle on the Neurovascular Safety of All-Inside Lateral Meniscus Repair: A Cadaveric Study. Arthroscopy. 2015 Nov;31(11):2138-44. doi: 10.1016/j.arthro.2015.04.100.

49. Chen N.C., Martin S.D., Gill T.J. Risk to the lateral geniculate artery during arthroscopic lateral meniscal suture passage. Arthroscopy. 2007;23:642-646.

50. Cuéllar A, Cuéllar R, Díaz Heredia J, Cuéllar A, García-Alonso I, Ruiz-Ibán MA. The allinside meniscal repair technique has less risk of injury to the lateral geniculate artery than the inside-out repair technique when suturing the lateral meniscus. Knee Surg Sports Traumatol Arthrosc. 2018 Mar;26(3):793-798. doi: 10.1007/s00167017-4490-1.

51. Westermann RW, Duchman KR, Amendola A, Glass N, Wolf BR. All-Inside Versus Inside-Out Meniscal Repair With Concurrent Anterior Cruciate Ligament Reconstruction: A Meta-regression Analysis. Am J Sports Med. 2017 Mar;45(3):719-724. doi: 10.1177/0363546516642220.

52. Moatshe G, Cinque ME, Godin JA, Vap AR, Chahla J, LaPrade RF. Comparable Outcomes After Bucket-Handle Meniscal Repair and Vertical Meniscal Repair Can Be Achieved at a Minimum 2 Years' Follow-up. Am J Sports Med. 2017 Nov;45(13):31043110. doi: $10.1177 / 0363546517719244$.

53. Uzun E, Misir A, Kizkapan TB, Ozcamdalli $M$, Akkurt S, Guney A. Evaluation of Midterm Clinical and Radiographic Outcomes of Arthroscopically Repaired
Vertical Longitudinal and Bucket-Handle Lateral Meniscal Tears. Orthop J Sports Med. $2019 \quad$ May 10;7(5):2325967119843203. doi: 10.1177/2325967119843203.

54. Ahn JH, Kim KI, Wang JH, Kyung BS, Seo $\mathrm{MC}$, Lee SH. Arthroscopic repair of buckethandle tears of the lateral meniscus. Knee Surg Sports Traumatol Arthrosc. 2015 Jan;23(1):205-10. doi: 10.1007/s00167013-2764-9.

55. Messner K, Gao J. The menisci of the knee joint. Anatomical and functional characteristics, and a rationale for clinical treatment. J Anat. 1998 Aug;193 ( Pt 2)(Pt 2):161-78. doi: 10.1046/j.14697580.1998.19320161.x.

56. Bedi A, Kelly N, Baad M, Fox AJ, Ma Y, Warren RF, Maher SA. Dynamic contact mechanics of radial tears of the lateral meniscus: implications for treatment. Arthroscopy. 2012 Mar;28(3):372-81. doi: 10.1016/j.arthro.2011.08.287.

57. Winkler PW, Wierer G, Csapo R, Hepperger C, Heinzle B, Imhoff AB, Hoser C, Fink C. Quantitative Evaluation of Dynamic Lateral Meniscal Extrusion After Radial Tear Repair. Orthop J Sports Med. 2020 Apr 10;8(4):2325967120914568. doi: $10.1177 / 2325967120914568$.

58. Alentorn-Geli E, Choi JH, Stuart JJ, Toth AP, Garrett WE, Taylor DC, Moorman CT 3rd. Inside-Out or Outside-In Suturing Should Not Be Considered the Standard Repair Method for Radial Tears of the Midbody of the Lateral Meniscus: A Systematic Review and Meta-Analysis of Biomechanical Studies. J Knee Surg. 2016 Oct;29(7):604612. doi: 10.1055/s-0035-1570113.

59. Matsubara H, Okazaki K, Izawa T, Tashiro $Y$, Matsuda S, Nishimura T, Nakanishi Y, Kawamura $H$, Iwamoto $Y$. New suture method for radial tears of the meniscus: biomechanical analysis of cross-suture and 
double horizontal suture techniques using cyclic load testing. Am J Sports Med. 2012 Feb;40(2):414-8. doi: 10.1177/0363546511424395.

60. Stender ZC, Cracchiolo AM, Walsh MP, Patterson DP, Wilusz MJ, Lemos SE. Radial Tears of the Lateral Meniscus-Two Novel Repair Techniques: A Biomechanical Study. Orthop J Sports Med. 2018 Apr 27;6(4):2325967118768086. doi: $10.1177 / 2325967118768086$.

61. Massey P, McClary K, Parker D, Barton RS, Solitro G. The rebar repair for radial meniscus tears: a biomechanical comparison of a reinforced suture repair versus parallel and cross-stitch techniques. J Exp Orthop. 2019 Aug 22;6(1):38. doi: 10.1186/s40634-019-0206-4.

62. Tsujii A, Yonetani Y, Kinugasa K, Matsuo T, Yoneda K, Ohori T, Hamada M. Outcomes More Than 2 Years After Meniscal Repair for Radial/Flap Tears of the Posterior Lateral Meniscus Combined With Anterior Cruciate Ligament Reconstruction. Am J Sports Med. 2019 Oct;47(12):2888-2894. doi: $10.1177 / 0363546519869955$.

63. Wu IT, Hevesi M, Desai VS, Camp CL, Dahm DL, Levy BA, Stuart MJ, Krych AJ. Comparative Outcomes of Radial and Bucket-Handle Meniscal Tear Repair: A Propensity-Matched Analysis. Am J Sports Med. 2018 Sep;46(11):2653-2660. doi: $10.1177 / 0363546518786035$.

64. Moulton SG, Bhatia S, Civitarese DM, Frank RM, Dean CS, LaPrade RF. Surgical Techniques and Outcomes of Repairing Meniscal Radial Tears: A Systematic Review. Arthroscopy. 2016 Sep;32(9):1919-25. doi: 10.1016/j.arthro.2016.03.029.

65. Schillhammer CK, Werner FW, Scuderi MG, Cannizzaro JP. Repair of lateral meniscus posterior horn detachment lesions: a biomechanical evaluation. Am J Sports Med. 2012;40(11):2604-2609.

66. Tang X, Marshall B, Wang JH, Zhu J, Li J, Smolinski $\mathrm{P}, \mathrm{Fu}$ FH. Lateral Meniscal Posterior Root Repair With Anterior Cruciate Ligament Reconstruction Better Restores Knee Stability. Am J Sports Med. 2019 Jan;47(1):59-65. doi: $10.1177 / 0363546518808004$.

67. Robinson JR, Frank EG, Hunter AJ, Jermin PJ, Gill HS. The Strength of Transosseous Medial Meniscal Root Repair Using a Simple Suture Technique Is Dependent on Suture Material and Position. Am J Sports Med. 2018;46(4):924-932. doi:10.1177/0363546517749807

68. Forkel P, Petersen W. Posterior root tear fixation of the lateral meniscus combined with arthroscopic $\mathrm{ACL}$ double-bundle reconstruction: technical note of a transosseous fixation using the tibial PL tunnel. Arch Orthop Trauma Surg. 2012 Mar;132(3):387-91. doi: 10.1007/s00402011-1429-8.

69. Pan F, Hua S, Ma Z. Surgical treatment of combined posterior root tears of the lateral meniscus and ACL tears. Med Sci Monit. 2015 May 11;21:1345-9. doi: 10.12659/MSM.892661.

70. Shelbourne KD, Roberson TA, Gray T. Longterm evaluation of posterior lateral meniscus root tears left in situ at the time of anterior cruciate ligament reconstruction. Am J Sports Med. 2011 Jul;39(7):1439-43. doi: 10.1177/0363546511398212.

71. Anderson L, Watts M, Shapter O, Logan M, Risebury M, Duffy D, Myers P. Repair of radial tears and posterior horn detachments of the lateral meniscus: minimum 2-year follow-up. Arthroscopy. 2010 Dec;26(12):1625-32. doi: 10.1016/j.arthro.2010.07.020. 
72. Ahn JH, Lee YS, Yoo JC, Chang MJ, Park SJ, Pae YR. Results of arthroscopic all-inside repair for lateral meniscus root tear in patients undergoing concomitant anterior cruciate ligament reconstruction. Arthroscopy. 2010 Jan;26(1):67-75. doi: 10.1016/j.arthro.2009.07.007.

73. Spang lii RC, Nasr MC, Mohamadi A, DeAngelis JP, Nazarian A, Ramappa AJ. Rehabilitation following meniscal repair: a systematic review. BMJ Open Sport Exerc Med. 2018;4(1):e000212. doi:10.1136/bmjsem-2016-000212

74. Koch M, Memmel C, Zeman F, Pfeifer CG, Zellner J, Angele $P$, Weber-Spickschen $S$, Alt V, Krutsch W. Early Functional Rehabilitation after Meniscus Surgery: Are Currently Used Orthopedic Rehabilitation Standards Up to Date? Rehabil Res Pract. 2020 Mar 29;2020:3989535. doi: $10.1155 / 2020 / 3989535$.

75. O'Donnell K, Freedman KB, Tjoumakaris FP. Rehabilitation Protocols After Isolated Meniscal Repair: A Systematic Review. Am J Sports Med. 2017 Jun;45(7):1687-1697. doi: $10.1177 / 0363546516667578$.

VanderHave KL, Perkins C, Le $M$. Weightbearing Versus Nonweightbearing After Meniscus Repair. Sports Health. 2015 Sep-Oct;7(5):399-402. doi: $10.1177 / 1941738115576898$. 